\begin{tabular}{|c|c|c|c|c|}
\hline Characteristic & $\begin{array}{l}\text { Clopidogrel } \\
(\mathrm{N}=13)\end{array}$ & $\begin{array}{l}\text { Prasugrel } \\
(\mathrm{N}=15)\end{array}$ & $\begin{array}{l}\text { Ticagrelor } \\
(\mathrm{N}=15)\end{array}$ & $\begin{array}{l}\text { P- } \\
\text { value }\end{array}$ \\
\hline Age (yrs) & 78À9.7 & 56À12.95 & $67.3 A ̀ 11.59$ & $<0.001$ \\
\hline Female & 6 & 3 & 4 & 0.302 \\
\hline Diabetes Mellitus & 0 & 4 & 2 & 0.127 \\
\hline Hypertension & 6 & 7 & 7 & 1 \\
\hline Current Smoker & 2 & 3 & 4 & 0.761 \\
\hline Ex Smoker & 4 & 8 & 4 & 0.271 \\
\hline Hyperlipidaemia & 4 & 5 & 3 & 0.691 \\
\hline $\begin{array}{l}\text { Familial History of } \\
\text { CAD }\end{array}$ & 6 & 9 & 8 & 0.765 \\
\hline
\end{tabular}

$\mathrm{CAD}=$ Coronary Artery Disease

Methods A single centre non-randomised study in patients presenting with STEMI. All patients gave informed consent. Enrolled patients were administered a loading dose of aspirin $300 \mathrm{mg}$ and then either clopidogrel $600 \mathrm{mg}$, prasugrel $60 \mathrm{mg}$ or ticagrelor $180 \mathrm{mg}$ prior to percutaneous coronary intervention. Platelet reactivity was measured using a Verify Now assay at 20 minutes post loading, first balloon inflation, 1 and 4 hours post loading. Results are expressed a P2Y12 reaction units (PRU). PRU $\geq 208$ indicates poor antiplatelet response. The effect of the three drugs over time were assessed using 2 way ANOVA. $\mathrm{P}<0.05$ was taken as a significant result.

Results A total of 43 STEMI patients were recruited to the study. Refer to Table 1 for baseline characteristics. PRU results for the 3 drugs at 20 minutes, balloon time, 60 minutes and 240 minutes were determined. Mean dose to balloon time was $26.8+12.7$. At balloon time, 20 minutes and 60 minutes none of the agents achieved a PRU $<208$. There was a significant difference over time between clopidogrel and ticagrelor $(\mathrm{P}=0.04)$, clopidogrel and prasugrel $\mathrm{P}<0.0001$ and no statistical difference was found when ticagrelor was compared with prasugrel over time (Figure 1).

Conclusion Clopidogrel in the context of STEMI does not provide adequate platelet inhibition as evidenced by PRU > 208 at all data collection time points, Both prasugrel and ticagrelor are superior to clopidogrel resulting in a significant reduction in the degree of platelet reactivity.

Although Prasugrel and ticagrelor are comparable in terms of inhibition of platelet activity at 20 minutes, balloon inflation, 1 hour and 4 hours, they still do not achieve the desired levels of platelet inhibition necessary during PPCI as demonstrated by PRU $>208$. It is possible that fast acting intravenous antiplatelet agents may have a role to play in achieving adequate platelet inhibition in the context of PPCI.

\section{HIGH-SENSITIVITY CARDIAC TROPONIN, PRE-TEST PROBABILITY AND THE DIAGNOSIS OF MYOCARDIAL INFARCTION: A PROSPECTIVE COHORT STUDY}

Andrew R Chapman*, Ala Noaman, Amar Vaswani, Mathew Gibbins, Megan Griffiths, Anoop SV Shah, Fiona Strachan, Atul Anand, Michelle D Souza, Gray Alasdair, David McAllister, David Newby, Nicholas Mills. University of Edinburgh; *Presenting Author

\subsection{6/heartjnl-2016-309890.112}

Background Cardiac troponin is integral to the diagnosis of myocardial infarction, but is elevated in many patients without acute coronary syndrome. We evaluate the impact of a clinical assessment of pre-test probability on the positive predictive value (PPV) of cardiac troponin for a diagnosis of myocardial infarction.

Methods In 1,054 consecutive patients undergoing blood sampling in the Emergency Department, cardiac troponin I was measured in excess serum using a high-sensitivity assay. Patients were classified as type 1 or type 2 myocardial infarction, or myocardial injury, and the prevalence determined in the entire cohort and in those where testing was requested by the attending physician. We determine the predicted PPV for type 1 myocardial infarction across different pre-test probabilities.

Results Cardiac troponin was requested in 136 patients identifying $15(11.3 \%)$ with type 1 myocardial infarction. Testing everyone identified 2 further patients with type 1 (17/1,054 [1.7\%]), but $127(12 \%)$ patients with type 2 myocardial infarction or myocardial injury. In patients selected for testing the PPV for type 1 myocardial infarction was 50.0\% (95\% confidence interval [CI] 32.8\%-67.2\%), falling to $13.6 \%(95 \%$ CI 8.0\%-19.7\%) when measured across the cohort. The PPV was heavily influenced by the pre-test probability (Figure 1). Irrespective of diagnosis, cardiac troponin was a independent predictor of death (hazard ratio 1.26 per 2 -fold increase, 95\% CI 1.06 to 1.49 ) (Figure 2).

Conclusions Cardiac troponin concentrations are elevated in one in eight patients in the Emergency Department. If testing

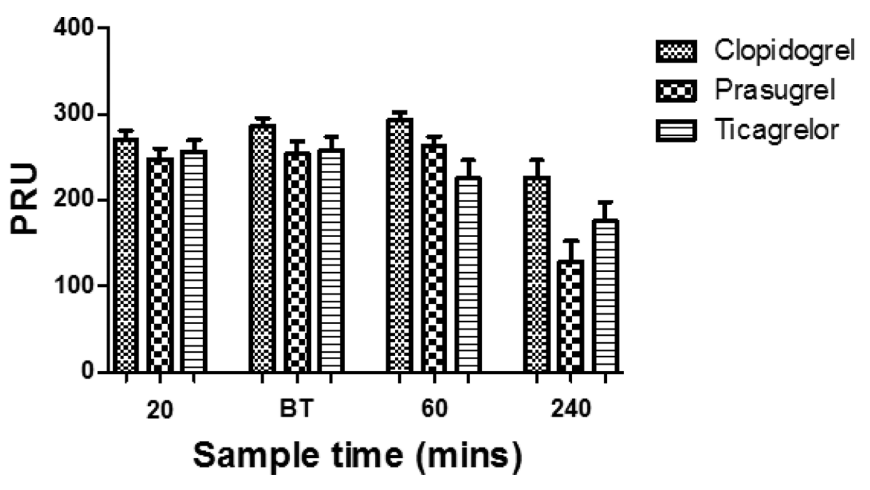

Abstract 111 Figure 1 The degree and time course of platelet inhibition (expressed as PRU) over time following the administration of clopidogrel, prasugrel or ticagrelor in STEMI patients. PRU $=$ P2Y12 Reactivity Units

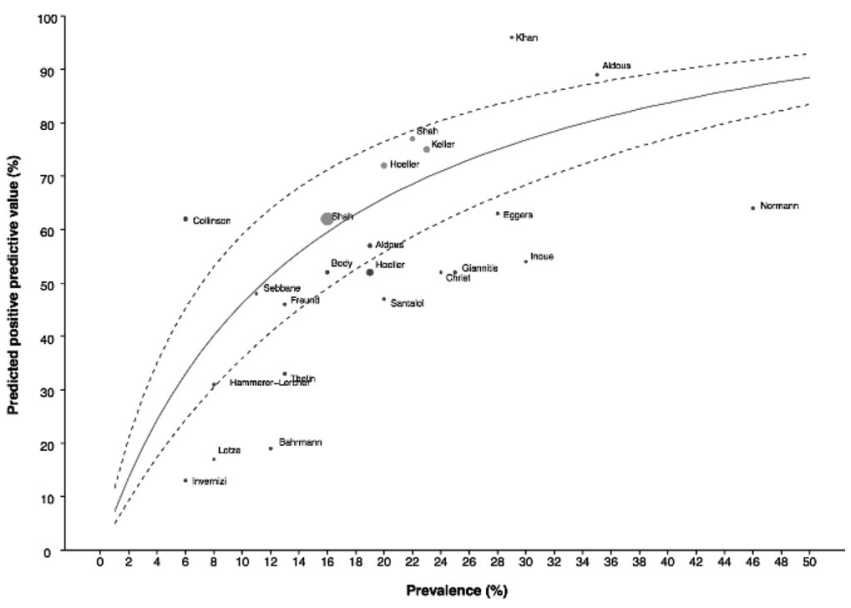

Abstract 112 Figure 1 


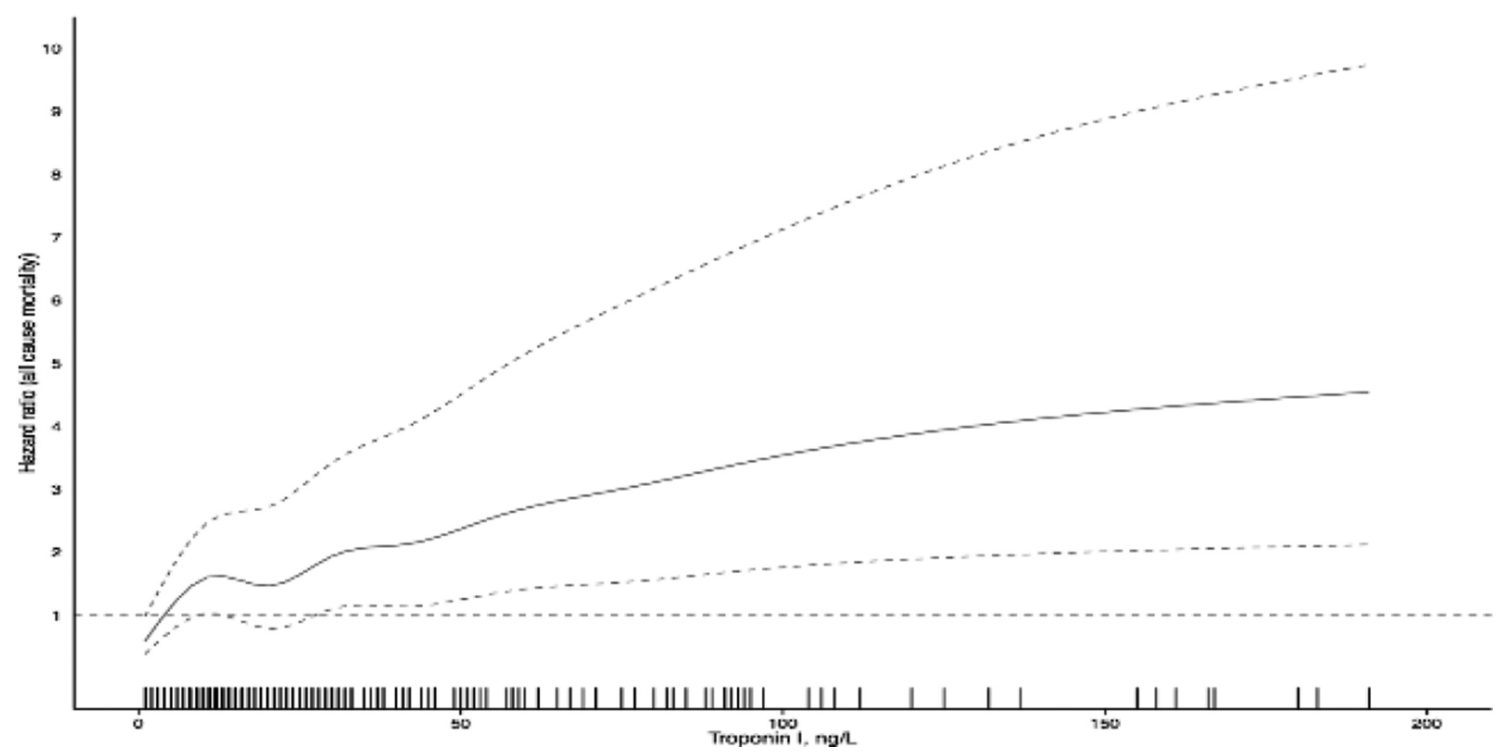

\section{Abstract 112 Figure 2}

is performed without an assessment of pre-test probability, interpretation of cardiac troponin will be challenging and may adversely impact on the diagnosis of acute myocardial infarction.

\section{LONG TERM OUTCOMES OF PATIENTS WITH TYPE 2 MYOCARDIAL INFARCTION OR MYOCARDIAL INJURY}

Andrew R Chapman*, Anoop SV Shah, Atul Anand, Fiona Strachan, David McAllister, David Newby, Nicholas Mills. University of Edinburgh; *Presenting Author

\subsection{6/heartjnl-2016-309890.113}

Background Type 2 myocardial infarction is defined as myocardial necrosis due to an imbalance in oxygen supply or demand, and is differentiated from myocardial injury by the presence of symptoms or signs of myocardial ischaemia. Both may occur in a wide range of cardiac and non-cardiac conditions and the long term consequences are uncertain.

Methods We identified all consecutive patients $(\mathrm{n}=2,122)$ presenting to a tertiary cardiac centre with cardiac troponin I concentrations above the diagnostic threshold of $50 \mathrm{ng} / \mathrm{L}$ irrespective of presenting complaint. Two cardiologists independently adjudicated the diagnosis of type 1 myocardial infarction, type 2 myocardial infarction or myocardial injury. The primary outcome was all-cause mortality. Secondary outcomes included subsequent hospitalisation for type 1 myocardial infarction, and cardiovascular or non-cardiovascular mortality. Incidence rates for primary and secondary outcomes were derived for patients stratified by the index diagnosis and cox proportional hazard models constructed to estimate risk of death or hospitalisation, adjusting for age and sex.

Results The adjudicated diagnosis was type 1 myocardial infarction in 1,171 patients (55.2\%), type 2 myocardial infarction in 429 patients $(20.2 \%)$ and myocardial injury in 522 patients (24.6\%; Table 1). During 8,809 patient years follow up there were 1,231 deaths (552 cardiovascular, 623 non-cardiovascular; Figure 1) and 307 patients were hospitalised for type 1 myocardial infarction. Patients with type 2 myocardial infarction were at higher risk of all-cause mortality compared to those with type 1 myocardial infarction (20.9 vs 8.7 events per 100 person years; hazard ratio [HR] 1.58, 95\% CI 1.371.82), but were at lower risk of subsequent hospitalisation for type 1 myocardial infarction (3.2 vs 4.3 events per 100 person years; HR 0.53 , 95\% CI 0.38-0.73). Survival in patients with myocardial injury was worse, even compared to those with type 2 myocardial infarction (27.6 vs 20.9 events per 100 person years; HR 1.17, 95\% CI 1.01-1.36).

Conclusions Patients with type 2 myocardial infarction and myocardial injury have a very poor prognosis compared to those with type 1 myocardial infarction. Whether worse outcomes are due to the presence of treatable cardiovascular disease or as a consequence of other co-morbid conditions requires further investigation.

Abstract 113 Table 1 Baseline characteristics of the study population stratified by index diagnosis

\begin{tabular}{|c|c|c|c|}
\hline & $\begin{array}{l}\text { Type } 1 \text { Myocardial } \\
\text { Infarction }(n==1171)\end{array}$ & $\begin{array}{l}\text { Type } 2 \text { Myocardial } \\
\text { Infarction }(n==429)\end{array}$ & $\begin{array}{l}\text { Myocardial } \\
\text { Injury ( } \mathrm{n}= \\
=522 \text { ) }\end{array}$ \\
\hline Age (mean (SD)) & $68(14.2)$ & $75(14.1)$ & $76(12.7)$ \\
\hline Male (\%) & $709(60.5)$ & $222(51.7)$ & $260(49.8)$ \\
\hline Smoker (\%) & $380(34)$ & $62(14.5)$ & 73 (14) \\
\hline $\begin{array}{l}\text { Diabetes } \\
\text { Mellitus (\%) }\end{array}$ & 185 (16.7) & $93(21.7)$ & $96(18.7)$ \\
\hline $\begin{array}{l}\text { Hypertension } \\
(\%)\end{array}$ & $533(48.2)$ & $254(59.3)$ & $303(58.9)$ \\
\hline $\begin{array}{l}\text { Hyperlipidaemia } \\
\text { (\%) }\end{array}$ & $539(48.6)$ & $177(41.5)$ & $202(39.5)$ \\
\hline $\begin{array}{l}\text { Family History } \\
(\%)\end{array}$ & $193(18.1)$ & $14(3.3)$ & $10(2.0)$ \\
\hline Previous MI (\%) & $231(23.9)$ & $109(26)$ & $107(20.9)$ \\
\hline $\begin{array}{l}\text { Previous } \mathrm{PCl} \\
(\%)\end{array}$ & $153(14.7)$ & $17(4)$ & $23(4.5)$ \\
\hline $\begin{array}{l}\text { Previous CABG } \\
(\%)\end{array}$ & $62(6.3)$ & $30(7.1)$ & $32(6.2)$ \\
\hline
\end{tabular}

